\title{
Oxidative stress, autophagy and pyroptosis in the neovascularization of oxygen-induced retinopathy in mice
}

\author{
SHUAI WANG $^{1 *}$, LI-YANG JI ${ }^{*}$, LI LI $^{2}$ and JING-MIN LI ${ }^{1}$ \\ ${ }^{1}$ Department of Ophthalmology, Second Affiliated Hospital of Dalian Medical University, Dalian, Liaoning 116023; \\ ${ }^{2}$ Department of Ophthalmology, Zaozhuang Municipal Hospital, Zaozhuang, Shandong 277000, P.R. China
}

Received May 7, 2018; Accepted November 8, 2018

DOI: $10.3892 / \mathrm{mmr} .2018 .9759$

\begin{abstract}
Retinal neovascularization (RNV) is a principal cause of visual impairment and blindness worldwide. The present study aimed to investigate how oxidative stress, autophagy and pyroptosis alter in RNV. The oxygen-induced retinopathy (OIR) model was established in C57BL/6J mice by exposing them to a high concentration of oxygen. RNV was clearly visible in the fundus images and was qualitatively analyzed by counting the number of neovascular endothelial cell nuclei at postnatal day 17 . Subsequently, the expression of vascular endothelial growth factor (VEGF)-A and hypoxia-inducible factor- $1 \alpha$ (HIF-1 $\alpha$ ) at the protein level were measured. Furthermore, oxidative stress was examined using dihydroethidium (DHE) staining, and NADPH oxidase (NOX) 1 and 4 in the retinas were detected using reverse transcription-quantitative polymerase chain reaction analysis. Additionally, immunostaining of microtubule associated protein 1 light chain $3 \alpha$ (LC3) was performed and the expression levels of the LC3, p62, autophagy protein (Atg)5, Atg7, Atg12, Beclin1, NOD-like receptor family pyrin domain-containing 3 (NLRP3), caspase-1, interleukin (IL)-1 $\beta$, pro-caspase-1 and pro-IL-1 $\beta$ proteins were determined using western blotting in order to detect pyroptosis and autophagic flux. Autophagosomes were also detected using transmission electron microscopy. The results revealed that VEGF-A and HIF-1 $\alpha$ protein expression levels, the DHE-positive area, and NOX1 and NOX4 mRNA expression levels were significantly increased in the OIR mice. Furthermore, increased levels of NLRP3, caspase-1, IL-1 $\beta$, pro-caspase-1 and pro-IL- $1 \beta$ proteins demonstrated that pyroptosis was activated. However, an accumulation of p62 and a reduction in the levels of LC3II/I and autophagosomes indicated that autophagic flux was compromised. Therefore, elevated levels
\end{abstract}

Correspondence to: Dr Jing-Min Li, Department of Ophthalmology, Second Affiliated Hospital of Dalian Medical University, 467 Zhongshan Road, Dalian, Liaoning 116023, P.R. China

E-mail: lijingmin218@126.com

*Contributed equally

Key words: retinal neovascularization, oxidative stress, autophagy, pyroptosis of reactive oxygen species and pyroptosis along with attenuated autophagy were demonstrated in the OIR mice. The combination of oxidative stress, pyroptosis and impaired autophagy may serve an important role in the pathophysiology of RNV and may be a potential target to prevent RNV.

\section{Introduction}

Retinal neovascularization (RNV) may be a result of numerous types of proliferative ischemic retinopathy, including retinopathy of prematurity (ROP), proliferative diabetic retinopathy (DR) and retinal vein occlusion (RVO). Although anti-vascular endothelial growth factor (VEGF) drugs, including ranibizumab and aflibercept, have exhibited potential as a treatment for RNV, an improved understanding of the mechanism underlying RNV is required in order to develop novel strategies for the prevention of neovascular retinal diseases.

Oxidative stress may be both a cause and a consequence of numerous types of vascular complications (1). The mammalian retina is an organ with high metabolic demand and reactive oxygen species (ROS) are generated through increased consumption of oxygen (2). NADPH oxidase (NOX) appears to be the only enzymatic source of ROS in the retina that is clearly involved in pathological neovascularization (3). Excessive levels of ROS cause endothelial dysfunction, which results in the loss of vascular cells and ischemia, and in turn triggers blood vessel growth (4).

VEGF is a factor in the direct stimulation of endothelial cell proliferation and tube formation in ischemia-induced RNV (5). Accumulating evidence supports the idea that ROS respond to VEGF and hypoxia-inducible factor-1 $\alpha$ (HIF-1 $\alpha)(6,7)$. Furthermore, NOX appears to be an important effector in redox signaling for the activation and signaling of HIF-1 $\alpha$ and VEGF (8).

Oxidative stress induces inflammation. Increased levels of ROS influence various physiological and pathological processes, including inflammation. An important mechanism of inflammation is the induction of inflammasomes, which activate caspase-1 and process inflammatory cytokines (9). This may result in pyroptosis, a form of inflammatory programmed cell death induced by inflammatory caspases (10). Pyroptosis releases the cytoplasmic contents from dying host cells, thereby providing potent signals to initiate an inflammatory cascade (11).

Increased generation of ROS in cells may induce the process of autophagy through transcriptional and post-transcriptional 
regulation (12). In general, ROS may be considered to be inducers of autophagy (13). Abnormal inflammation disrupts cellular homeostasis (14). Exposure to a highly inflammatory environment causes severe damage to tissues. Damaged cellular components may be digested by autophagy, a process of 'self-eating' that is induced by oxidative stress and hypoxia in order to maintain homeostasis (15).

In the present study, the role of oxidative stress, autophagy and pyroptosis in retinal angiogenesis was investigated in a mouse model of OIR. The results suggested that oxidative stress is potentially implicated in the development of retinal vasculature through upregulation of pyroptosis and downregulation of autophagy.

\section{Materials and methods}

Animals and oxygen-induced retinopathy (OIR) model (16). Pregnant female C57BL/6 mice at 8-10 weeks of age and 20-25 g post-mating were purchased from Jackson Laboratory (Bar Harbor, ME, USA). They were randomly divided into two groups: A normoxia group [wild-type (WT) group; $n=32$ ] and an oxygen-exposed group (ROP group; $n=30$ ). Postnatal day 7 (P7) mice and their mothers were placed in conditions of hyperemia ( $75 \pm 5 \%$ oxygen) for 5 days, and were subsequently removed and placed in air (21\% oxygen) for an additional 5 days. The incubator temperature was maintained at $21 \pm 2^{\circ} \mathrm{C}$ with 45-60\% humidity, on a 12/12 h day/night cycle with food and water ad libitum. Oxygen levels were checked using a CY-12C portable oxygen measuring instrument (AIPU Instruments, Ltd., Hangzhou, China). The mice in the normoxia group were maintained in normal room air from birth until P17. Following fundus photography, the mice were sacrificed by an overdose of pentobarbital $(100 \mathrm{mg} / \mathrm{kg}$; intra-peritoneal injection; Sigma-Aldrich; Merck KGaA, Darmstadt, Germany). The eyes were removed and prepared for further histological and molecular analysis. All investigations were approved by the Animal Care and Institutional Ethics Committee of Dalian Medical University (no. LCKY2016-31) and conformed to the US National Institutes of Health (Bethesda, MD, USA) Guide for the Care and Use of Laboratory Animals and the ARRIVE guidelines (17).

Fundus photography. Mydriatic eye drops (5\% tropicamide) were given to dilate the pupil in order to obtain a better view of the ocular fundus of wild-type and OIR mice on P17, following general anesthesia. Once the pupil was dilated, a fundus camera (OPTO-RIS; Optoprobe Science, Ltd., Richmond, BC, Canada) was used to view the inner surfaces of the eyes in a dark room. The appearance of the fundus was documented.

Hematoxylin and eosin $(H \& E)$ staining. The eyes were fixed with $40 \mathrm{~g} / 1$ paraformaldehyde in PBS overnight at $4^{\circ} \mathrm{C}$ and subsequently embedded in paraffin. Sections (5- $\mu$ m-thick) of the whole eye were stained with $\mathrm{H} \& \mathrm{E}$ at room temperature, according to a standard method. The nuclei of new vessels extending from the retina to the vitreous were counted in six sections at magnification $\mathrm{x} 400$ in each group using an Olympus fluorescence microscope (Olympus Corporation, Tokyo, Japan). Acquired images were processed using Image J version 1.48 (National Institutes of Health).
Dihydroethidium (DHE) staining. The ROS levels were measured in situ with the fluorescent probe DHE. Fresh frozen eye sections $\left(-26^{\circ} \mathrm{C} ; 6-\mu \mathrm{m}\right.$ thickness) were incubated with DHE ( $1 \mu \mathrm{M}$ in PBS) for $30 \mathrm{~min}$ at $37^{\circ} \mathrm{C}$. Digital images were taken of 10 random fields from each sample using an Olympus fluorescence microscope at magnification x400 (Olympus Corporation), and the positive areas were analyzed using Image $\mathrm{J}$ analysis software.

Western blotting. Pups from all groups were sacrificed on P17. Retinas from three mice mixed for one sample, total proteins were extracted using radioimmunoprecipitation assay buffer (Beyotime Institute of Biotechnology, Haimen, China). Equal amounts of protein $(40 \mu \mathrm{g})$ by bicinchoninic acid protein assay were loaded onto $12 \%$ SDS-PAGE gels. Following electrophoresis and wet transfer to a polyvinylidene difluoride (PVDF) membrane, the membrane was blocked in 5\% skim milk in TBS with Tween-20 (TBST) at room temperature for $1 \mathrm{~h}$. Immunostaining was conducted using antibodies against VEGF-A (cat. no. ab52917; 1:500), caspase-1 (cat. no. ab1872; 1:1,000), pro-caspase-1 (cat. no. ab179515; 1:1,000), interleukin (IL)-1 $\beta$ (cat. no. ab2105; 1:500), pro-IL-1 $\beta$ (cat. no. ab2105; 1:500), microtubule associated protein 1 light chain $3 \alpha$ (LC3; cat. no. ab48394; 1:500)

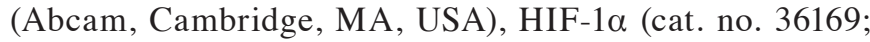
1:1,000), autophagy protein (Atg)5 (cat. no. 12994; 1:1,000), Atg7 (cat. no. 8558; 1:1,000), Atg12 (cat. no. 4180; 1:1,000), Beclin1 (cat. no. 3495; 1:1,000), p62 (cat. no. 23214; 1:1,000), NOD-like receptor family pyrin domain-containing 3 (NLRP3; cat. no. 15101; 1:1,000) and GAPDH (cat. no. 5174; 1:1,000; Cell Signaling Technology, Inc, Danvers, MA, USA) at $4^{\circ} \mathrm{C}$ overnight. The blots were subsequently incubated with horseradish peroxidase-conjugated secondary antibody (cat. no. A0208; 1:1,000; Beyotime Institute of Biotechnology) at room temperature for $2 \mathrm{~h}$. All blots were developed using a chemiluminescence system (Fluor Chem M System; ProteinSimple, San Jose, CA, USA) and signal intensities were analyzed with a Gel-pro 4.5 Analyzer (Media Cybernetics, Inc., Rockville, MD, USA). After measuring the intensity of each band by densitometry using the image processing software Image $\mathrm{J}$, the relative intensities were calculated by normalizing to GAPDH from the corresponding sample.

Reverse transcription-quantitative polymerase chain reaction $(R T-q P C R)$. Total RNA was extracted from retinas using TRIzol ${ }^{\circledR}$ reagent (Invitrogen; Thermo Fisher Scientific, Inc., Waltham, MA, USA), according to the manufacturer's protocol. The first strand cDNA was synthesized from 1-2 $\mu \mathrm{g}$ total RNA via oligo (dT)-primed RT (priming for $5 \mathrm{~min}$ at $25^{\circ} \mathrm{C}$; RT for $20 \mathrm{~min}$ at $46^{\circ} \mathrm{C}$; RT inactivation for $1 \mathrm{~min}$ at $95^{\circ} \mathrm{C}$; iScriptcDNA synthesis kit; Bio-Rad Laboratories, Inc., Hercules, CA, USA). The primers were designed using Primer-BLAST, based on the published GenBank sequence (https://blast.ncbi.nlm.nih.gov/Blast.cgi; https://www.ncbi. nlm.nih.gov/genbank/). All primer pairs are listed in Table I. RT-qPCR with SYBR (Takara Bio, Inc., Osaka, Japan) was performed with an ABI 7500 Fast Real-Time PCR System (Applied Biosystems; Thermo Fisher Scientific, Inc.) and quantified by $2^{-\Delta \Delta \mathrm{Cq}}$ method (18). 
Table I. Sequences of polymerase chain reaction primers.

\begin{tabular}{lll}
\hline Gene & \multicolumn{1}{c}{ Forward primer $\left(5^{\prime}-3^{\prime}\right)$} & \multicolumn{1}{c}{ Reverse primer $\left(5^{\prime}-3^{\prime}\right)$} \\
\hline NOX1 & CAGTTATTCATATCATTGCACACCTATTT & CAGAAGCGAGAGATCCATCCA \\
NOX4 & GCACGCTGTTGATTTTATGG & GCGAGGCAGGAGAGTCAGTA \\
GAPDH & GTGTTTCCTCGTCCCGTAGA & AATCTCCACTTTGCCACTGC
\end{tabular}

NOX, NADPH oxidase.

Immunofluorescence staining. Eyes were fixed with $40 \mathrm{~g} / 1$ paraformaldehyde in PBS overnight at $4^{\circ} \mathrm{C}$ and infiltrated with $25 \%$ sucrose. The cryosections were blocked with $1 \%$ bovine serum albumin (cat. no. HZB0148; Sigma-Aldrich; Merck $\mathrm{KGaA}$ ) at room temperature for $0.5 \mathrm{~h}$. Following incubation with anti-LC3 antibody (cat. no. ab48394; 1:500; Abcam) at $4^{\circ} \mathrm{C}$ overnight, sections were incubated with anti-rabbit IgG (cat. no. A0453; Alexa Fluor 555; 1:100; Beyotime Institute of Biotechnology) at $4^{\circ} \mathrm{C}$ for $1 \mathrm{~h}$. DAPI (1:10,000; Sigma-Aldrich; Merck KGaA) was used to label the nucleus at a concentration of $5 \mu \mathrm{g} / \mathrm{ml}$ at room temperature for $5 \mathrm{~min}$. Fluorescence images were acquired using a confocal Olympus microscope at magnification, $\mathrm{x} 400$ (Olympus Corporation).

Transmission electron microscopy. Eyes were isolated and fixed in $2.5 \%$ glutaraldehyde at $4{ }^{\circ} \mathrm{C}$ overnight and $1 \%$ osmium tetroxide at room temperature successively, followed by dehydration in ethanol. Following incubation in acetone for $20 \mathrm{~min}$, the eyes were treated with $50 \%(1 \mathrm{~h}), 75 \%(3 \mathrm{~h})$ and $100 \%$ (overnight) epoxy resin and heated at $70^{\circ} \mathrm{C}$ overnight. The embedded eyes were sliced to ultrathin sections $(70 \mathrm{~nm})$ using an MT-5000 Sorvall microtome (Sorvall; Thermo Fisher Scientific, Inc.). Sections were stained with $3 \%$ uranyl acetate and $3 \%$ lead citrate for $15 \mathrm{~min}$ at room temperature and were visualized with a transmission electron microscope system.

Statistical analysis. All experiments were performed at least three times, and the results of one representative experiment are presented as the mean \pm standard deviation. For comparisons between two groups, the independent sample t-test (normally distributed) or Mann-Whitney test (non-normally distributed) was used. $\mathrm{P}<0.05$ was considered to indicate a statistically significant difference. Analyses were performed using SPSS 22.0 (IBM Corp., Armonk, NY, USA).

\section{Results}

$R N V$ is clearly generated in OIR mice. To investigate whether the mouse model of OIR was successfully established, a fundus camera was used to capture images of the retina. The fundus images revealed that neovascular tufts were successfully generated in the OIR mice. Large numbers of pale neovascular vessels had grown into the vitreous cavity in the OIR mice (Fig. 1A) and the number of neovascular nuclei was significantly greater compared with that in the WT mice (Fig. 1B and C).
Angiogenesis is activated in the retina of OIR mice. The effect on retinal angiogenesis in OIR mice was determined. When OIR had been achieved, the protein expression levels of VEGF-A and those of its upstream regulatory molecule HIF-1 $\alpha$ were significantly increased in the retinas of the OIR mice compared with the WT control (Fig. 2). These results indicated that angiogenesis was activated, via the HIF-1 $\alpha /$ VEGF-A signaling pathway, in this model.

Retinal oxidative stress is increased in OIR mice. ROS is one of the principal triggers of blood vessel growth. To assess the alteration in the levels of ROS in the OIR mice in the present study, retinal sections were assessed using DHE staining. The retinas from the OIR mice exhibited a significant increase in the intensity of DHE staining (Fig. 3A and B). A major source of ROS in endothelial cells is NOX. In particular, the isoforms NOX1 and NOX4 are known to be involved in the generation of retinal $\operatorname{ROS}(19,20)$. The mRNA expression levels of NOX1 and NOX4 were determined in the present study using RT-qPCR analysis. As with the levels of ROS, the mRNA expression levels of NOX1 and NOX4 were significantly increased in the retinas from the OIR mice compared with the WT control mice (Fig. 3C and D).

Pyroptosis is activated in the retinas of OIR mice. In order to elucidate the involvement of inflammasomes in OIR, the expression of NLRP3 was analyzed using western blotting. There was a significant increase in the levels of NLRP3 expression in the OIR mice compared with the WT control mice (Fig. 4). As oxidative stress activates inflammasome-caspase-1-IL-1 $\beta$ signaling, whether caspase-1 is activated in the hypoxia situation of the retinas was investigated. The protein levels of caspase-1 and pro-caspase-1, which were detected using western blotting, were significantly increased in the OIR mice compared with the WT control mice (Fig. 4). Furthermore, the pro-inflammatory cytokines IL-1 $\beta$ and pro-IL-1 $\beta$ also exhibited an increase at the protein level (Fig. 4).

Autophagic flux is reduced in the retinas of OIR mice. In recent years, autophagy has been identified as a biological process associated with inflammation in different cellular contexts, as it targets inflammasome components for degradation (21). On that basis, it was hypothesized that autophagy may be involved in OIR. As lipidation of LC3 is a useful marker of autophagy, LC3 was analyzed using immunofluorescence and western blotting. The expression levels of Atg5, Atg7, Atg12 and Beclin1 were also determined using western 


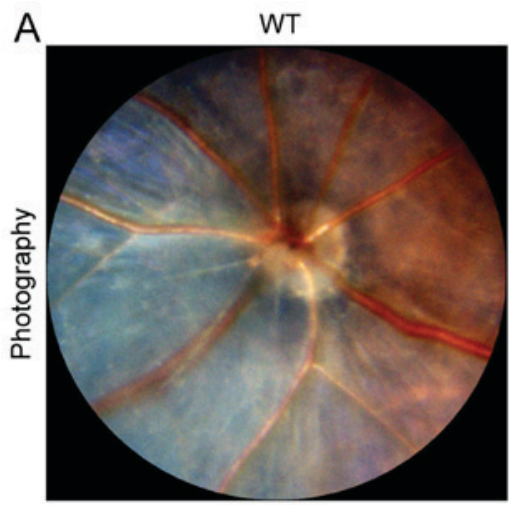

B

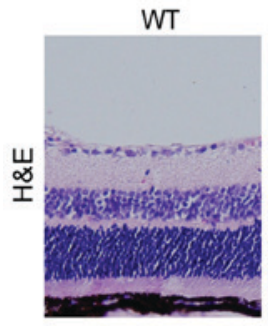

OIR

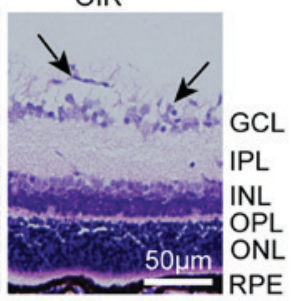

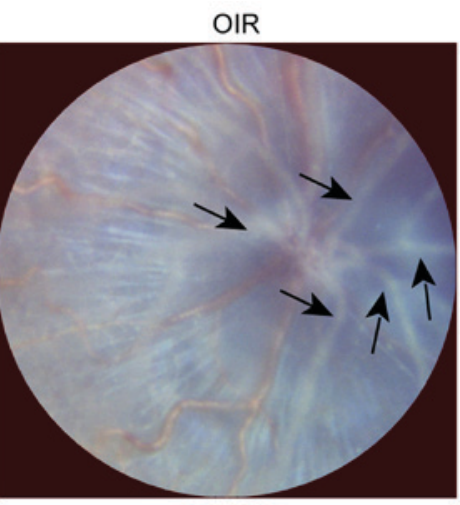

C

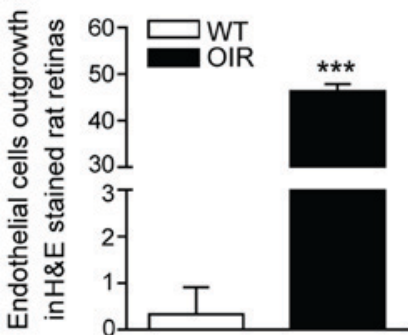

Figure 1. RNV is generated in OIR mice. (A) Fundus photography facilitated visualization of the inner surfaces of the eyes of the WT and OIR mice. The representative images indicate the large number of pale neovascular vessels that had grown into the vitreous cavity in the OIR mice, compared with the WT mice. (B) Representative $\mathrm{H} \& \mathrm{E}$ staining of retinal sections. A large number of the neovascular nuclei were present beyond the internal limiting membrane in the OIR mice, while there were only a few neovascular nuclei in the WT mice. (C) The number of outgrowth endothelial cells in the retinas was calculated using Image-Pro Plus software. The number of neovascular nuclei in the OIR mice was significantly greater compared with that of the control mice. Data are presented as the mean \pm standard deviation of the mean ( $\mathrm{n}=6$ mice per group). ${ }^{* * *} \mathrm{P}<0.001$ vs. WT mice. GCL, ganglion cell layer; IPL, inner plexiform layer; INL, inner nuclear layer; OPL, outer plexiform layer; ONL, outer nuclear layer; RPE, retinal pigment epithelium. H\&E, hematoxylin and eosin; OIR, oxygen-induced retinopathy; WT, wild-type.

blotting. The immunostaining identified that the distribution of LC3 was reduced in the OIR mice compared with the WT control mice (Fig. 5A). Furthermore, transmission electron micrographs of the retinas demonstrated that there were fewer autophagosomes in the OIR mice compared with the WT control mice (Fig. 5B). There was a significant reduction in the expression levels of LC3II/I, Atg5, Atg7, Atg12 and Beclin1 in the retinas from the OIR mice compared with the WT control mice (Fig. 5C and D). However, a reduction in LC3II/I, which is expressed in autophagosomes, does not always confirm autophagic flux (22). Thus, the protein levels of p62, a selective substrate of autophagy, were also analyzed. The results demonstrated that there was an increase in the p62 protein levels in the OIR mice compared with the WT control mice (Fig. 5C and D), indicating that autophagic flux was blocked in the retinas of the OIR mice.

\section{Discussion}

Aberrant RNV is a principal cause of visual impairment and blindness in the context of neovascular ocular diseases, including ROP, DR and RVO. Among them, ROP is a major cause of childhood blindness worldwide (23). In certain countries, particularly those with middle and low incomes, the incidence of ROP is $>40 \%$ due to the increasing survival rate of premature infants and limited fundoscopic follow-up evaluation (24). Although anti-VEGF and laser ablation therapies may be successful, the long-term efficacy remains uncertain. A clear understanding of the mechanisms underlying RNV generation is required in order to develop novel therapeutic alternatives.
A
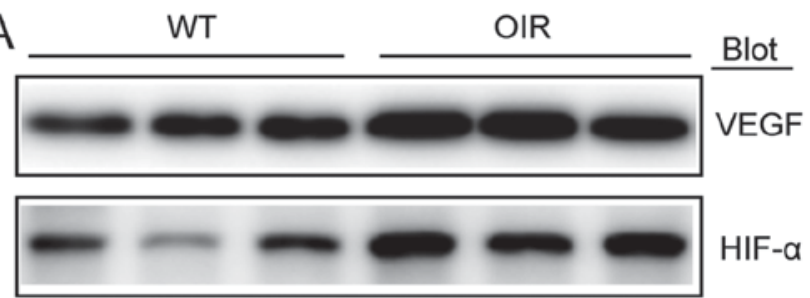

HIF-a

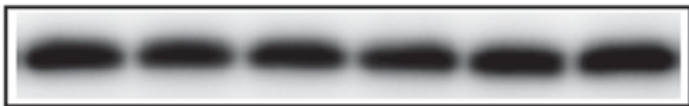

GAPDH

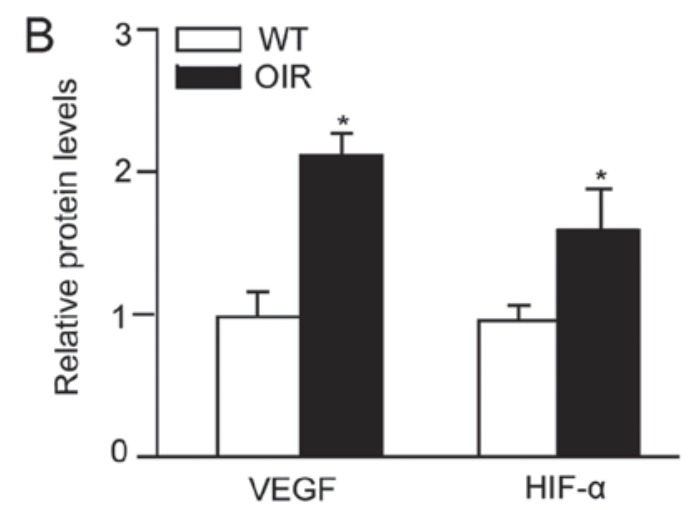

Figure 2. VEGF-A expression is activated in the retinas of OIR mice. (A) Immunoblot analysis of the protein expression levels of VEGF-A and HIF-1 $\alpha$ in the retinas. (B) Quantification revealed an increase in the expression levels of VEGF-A and HIF-1 $\alpha$ in the retinas of the OIR mice compared with WT mice. The relative protein expression level was normalized to GAPDH ( $\mathrm{n}=3$ mice per group). Data are presented as the mean \pm standard deviation of the mean. ${ }^{*} \mathrm{P}<0.05$ vs. WT mice. OIR, oxygen-induced retinopathy; WT, wild-type; VEGF, vascular endothelial growth factor; HIF-1 $\alpha$, hypoxia inducible factor- $1 \alpha$. 

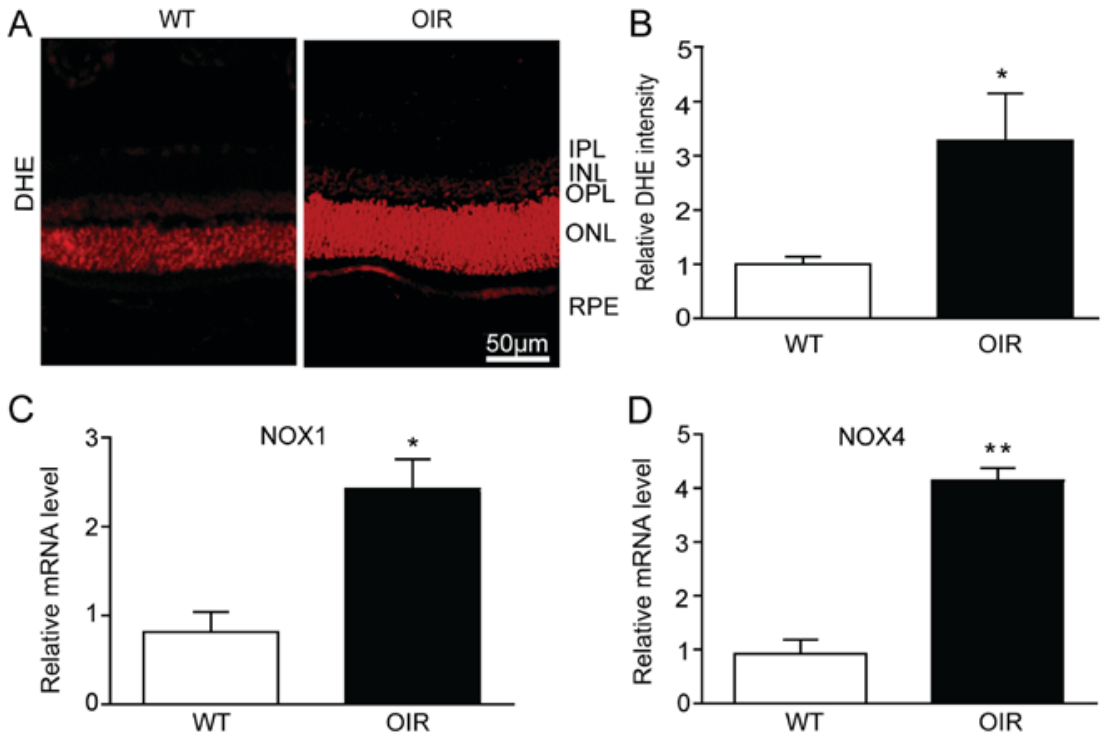

Figure 3. Retinal oxidative stress is increased in OIR mice. (A) The reactive oxygen species levels in the retinas of the WT and OIR mice were detected using the DHE fluorescent probe and fluorescent images of the retinas were visualized with a fluorescence microscope. Scale bar, $50 \mu \mathrm{m}$. (B) Quantification of DHE intensity in the retinas ( $\mathrm{n}=6$ mice per group). The retinas were harvested for RNA extraction and qPCR detection. (C) NOX1 and (D) NOX4 were upregulated at the mRNA level in the retinas from the OIR mice compared with the WT mice. The relative mRNA expression levels were normalized to GAPDH (n=6 mice per group). Data are presented as the mean \pm standard deviation of the mean. ${ }^{*} \mathrm{P}<0.05,{ }^{* *} \mathrm{P}<0.01$ vs. WT mice. IPL, inner plexiform layer; INL, inner nuclear layer; OPL, outer plexiform layer; ONL, outer nuclear layer; RPE, retinal pigment epithelium; OIR, oxygen-induced retinopathy; WT, wild-type; VEGF, vascular endothelial growth factor; DHE, dihydroethidium.

A
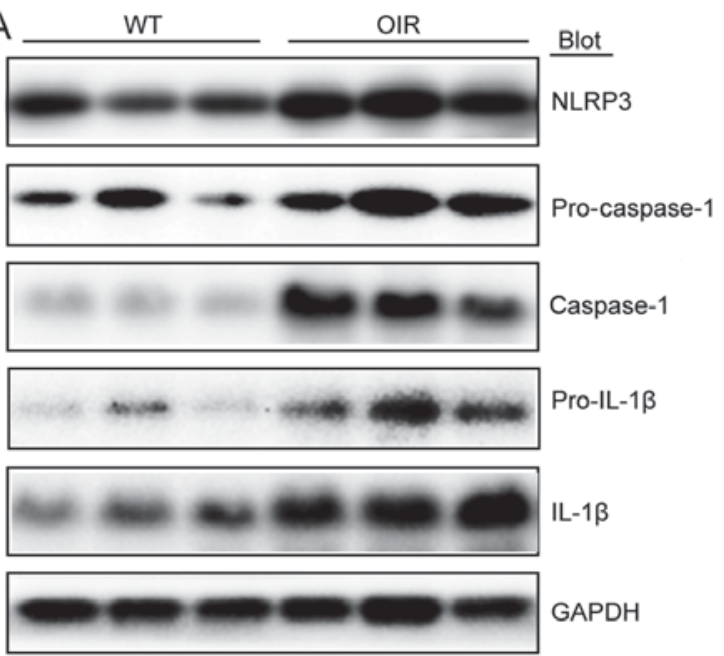

B
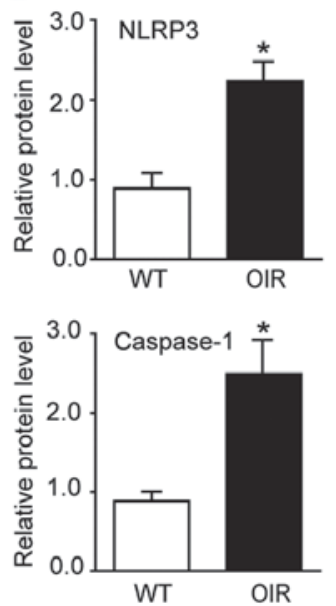
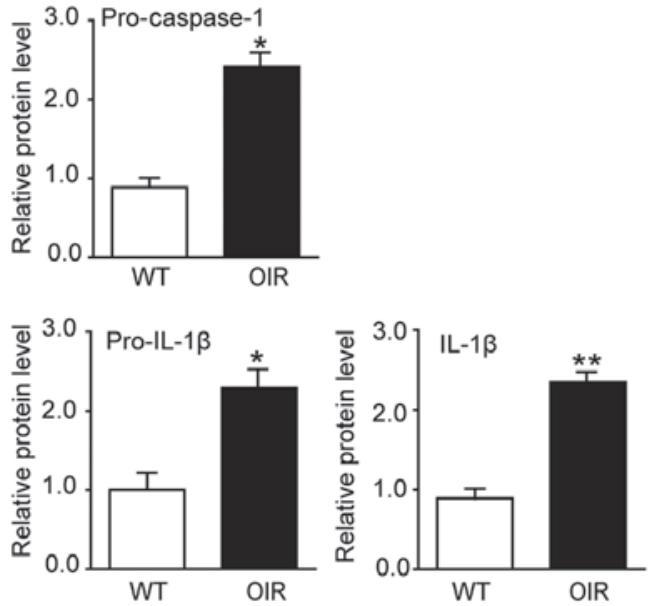

Figure 4. Inflammasome activity is increased in the retinas of OIR mice. (A) Immunoblot analysis of the protein expression levels of NLRP3, caspase-1, IL-1 $\beta$, pro-caspase-1 and pro-IL-1 $\beta$ in the retinas. (B) Quantification revealed an increase in the expression levels of NLRP3, caspase-1, IL-1 $\beta$, pro-caspase-1 and pro-IL-1 $\beta$ in the retinas of the OIR mice compared with the WT mice. The relative protein expression levels were normalized to GAPDH ( $\mathrm{n}=6$ mice per group). Data are presented as the mean \pm standard deviation of the mean. ${ }^{*} \mathrm{P}<0.05,{ }^{* *} \mathrm{P}<0.01 \mathrm{vs}$. WT mice. IL, interleukin; NLRP3, NOD-like receptor family pyrin domain-containing 3; OIR, oxygen-induced retinopathy; WT, wild-type.

RNV is stimulated by one or more angiogenic factors that are released by the retina under ischemic or hypoxic conditions, including VEGF, HIFs and cytokines (25). VEGF is an important pathogenic factor that stimulates endothelial cell proliferation and tube formation and mediates ischemia-induced RNV (5). The expression of retinal VEGF is increased under stress conditions in order to generate neovascularization (26). Therefore, understanding of the regulation of VEGF may aid investigations regarding the role of transcriptional regulators in RNV. In the present study, increased expression of VEGF-A and HIF-1 $\alpha$ in the OIR mice suggested that the angiogenesis in OIR mice is generated via the HIF-1 $\alpha /$ VEGF-A signaling pathway.

Oxidative stress occurs when the generation of pro-oxidants or ROS exceeds the capacity of endogenous antioxidants. ROS, including the superoxide anion $\left(\mathrm{O}_{2}^{-}\right)$, hydroxyl radical $(\mathrm{OH})$, hydrogen peroxide $\left(\mathrm{H}_{2} \mathrm{O}_{2}\right)$ and singlet oxygen $\left({ }^{1} \mathrm{O} 2\right)$, are primarily generated by mitochondria and NOX. The NOX family members serve a vital role in multiple cellular biological processes, including signal transduction, migration and proliferation (27). Furthermore, NOX appears to be the only enzymatic source of ROS in the retina that is clearly involved 
A

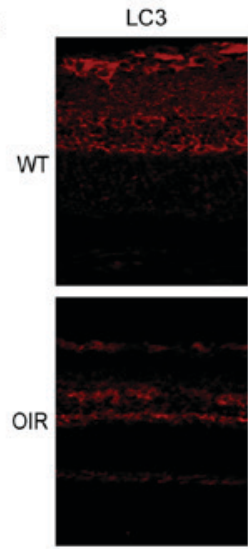

B

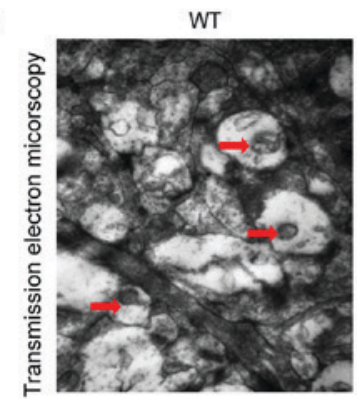

DAPI
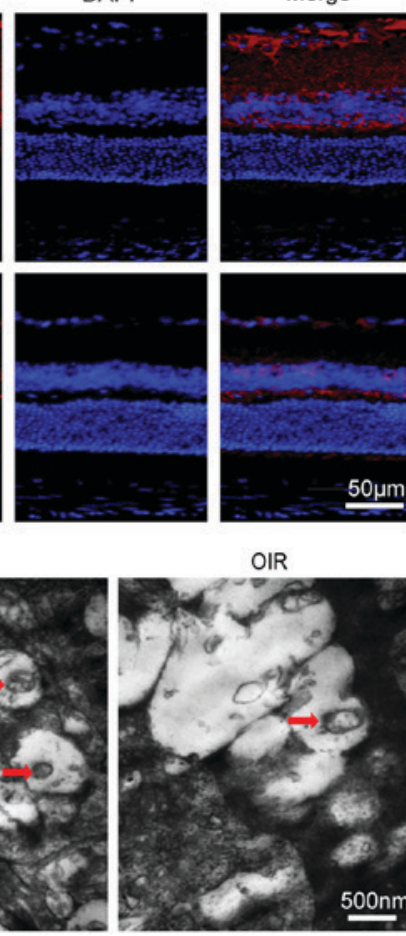

Merge
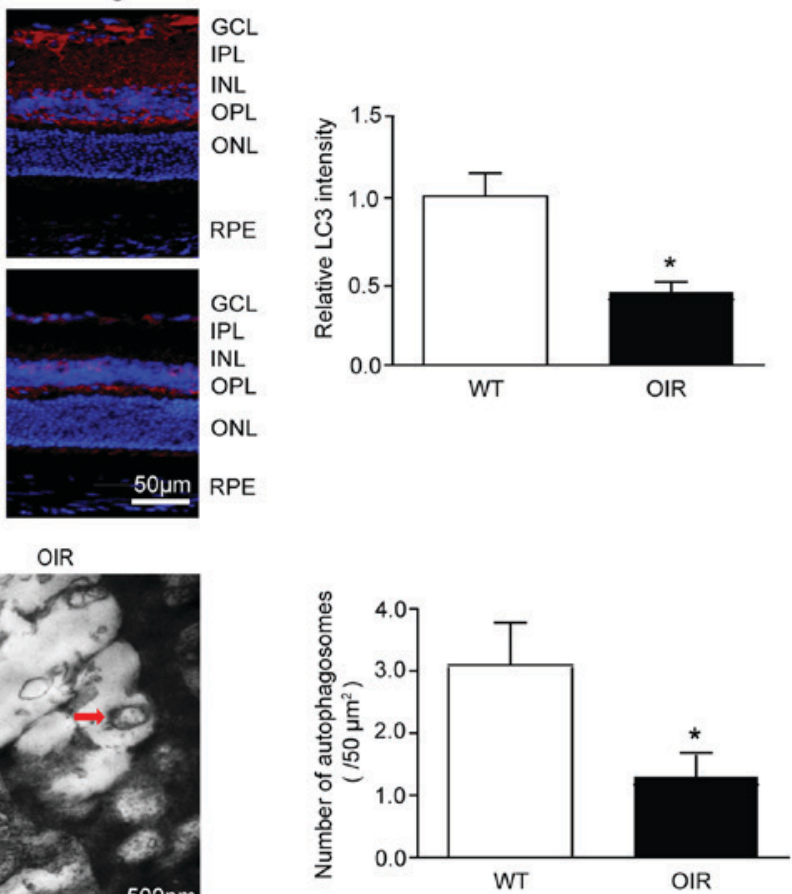

C
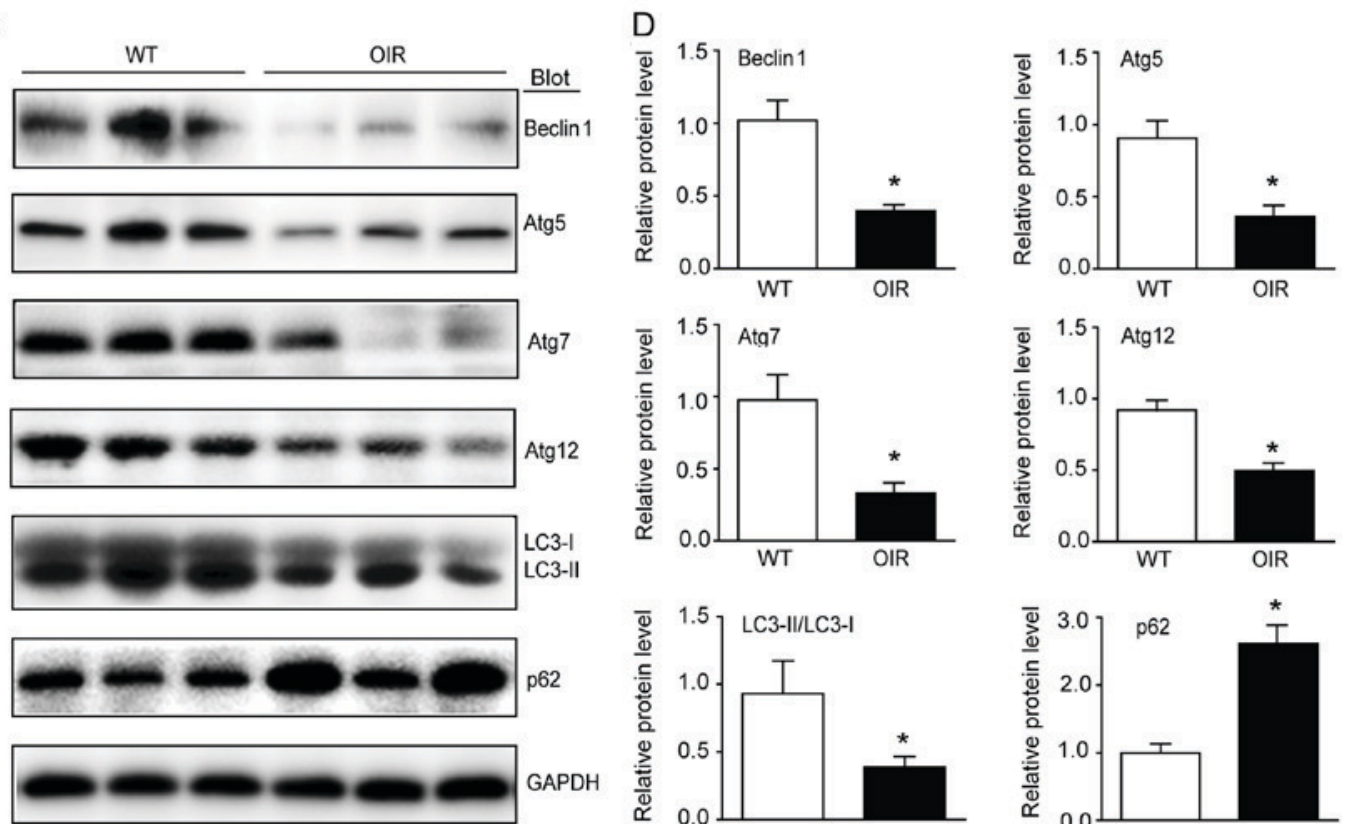

GAPDH
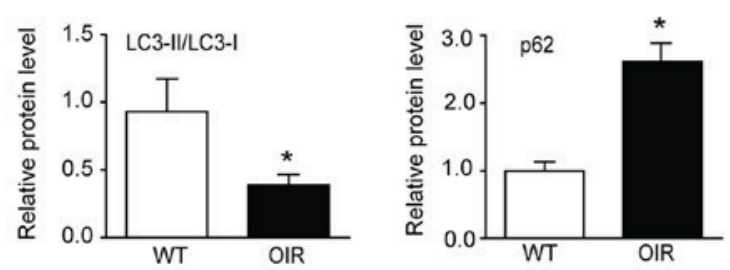

Figure 5. Autophagic flux is reduced in the retinas of OIR mice. (A) Immunostaining of the LC3 (red) in the retinal sections (left) and quantification of fluorescence intensity (right; $\mathrm{n}=6$ mice per group). Nuclei were counterstained with DAPI (blue). Scale bar, $50 \mu \mathrm{m}$. (B) Transmission electron micrographs and quantification of the autophagosomes in retinas. Scale bar, $500 \mathrm{~nm}$. (C) Immunoblot analysis of the protein expression levels of Beclin1, Atg5, Atg7, Atg12, LC3 and p62 in the retinas. (D) Quantification of the protein bands ( $n=6$ mice per group). GAPDH as an internal control. Data are the mean \pm standard deviation of the mean. ${ }^{*} \mathrm{P}<0.05$ vs. WT mice. OIR, oxygen-induced retinopathy; GCL, ganglion cell layer; IPL, inner plexiform layer; INL, inner nuclear layer; OPL, outer plexiform layer; ONL, outer nuclear layer; RPE, retinal pigment epithelium; Atg, autophagy protein; LC3, microtubule associated protein 1 light chain $3 \alpha$; WT, wild-type.

in pathological neovascularization (3). NOX1 and NOX4 are the major isoforms of NOX that are highly expressed in retinal endothelial cells of mice following stress $(19,20)$. In hypoxic environments, increased amounts of ROS are generated to mediate the hypoxia-induced cellular response (28). In the present study, DHE staining, NOX1 and NOX4 were detected in order to demonstrate that hypoxia stimulates the accumulation of ROS in the retinas of the OIR mice.
Increased generation of intracellular ROS may induce programmed cell death, such as autophagy and pyroptosis, via execution by lysosomal proteases or caspases (12). Pro-IL-1 $\beta$ and pro-caspase-1 are stored in secretory lysosomes, where they await an exocytosis-inducing stimulus; in the absence of such a stimulus, these molecules may undergo lysosomal degradation (29). IL-1 $\beta$ activation and release require the synthesis of pro-IL-1 $\beta$ and caspase-1 activation. Pyroptosis is a 
pro-inflammatory type of programmed cell death that is triggered by increased oxidative stress and the subsequent activation of inflammasome-caspase-1-IL-1 $\beta$ signaling, resulting in tissue injury (30). NOX-mediated oxidative stress is an initial signal that induces inflammasome activation. The inflammasome is a large supramolecular complex that is largely composed of dimers of the adaptor protein apoptosis-associated speck-like protein containing a CARD. In particular, the generation of ROS via NOX causes thioredoxin-interacting protein to associate with NLRP3, which facilitates inflammasome formation (31). Subsequently, caspase-1 is activated by the inflammasome and promotes the cascade and release of the highly pro-inflammatory cytokine IL-1 $\beta$. Previous studies have suggested that pyroptosis may be functionally linked with autophagy. A recent study demonstrated that reactive oxygen species induce NLPR3 inflammasomes mediated pyroptosis in the intestinal cells (32). It has been demonstrated that the inhibition of autophagy stimulates pneumococcus-induced pyroptosis and protects microglial cells against pyroptosis (33). The present study revealed an increase in the protein expression levels of NLRP3, caspase-1 and IL-1 $\beta$ in OIR mice, which indicated that pyroptosis is activated during vascularization of the retina.

Autophagy, also termed 'self-eating', is a highly sensitive cellular process that is induced in response to a wide range of stresses, including starvation, hypoxia, cytotoxicity and infection, in order to maintain homeostasis (15). Prolonged exposure to high levels of ROS and inflammasomes represents a stressful environment. A number of studies have suggested that ROS induce autophagy as upstream modulators $(34,35)$. In RPE cells under oxidative stress, autophagy is increased and autophagic flux is reduced, which are stimulated by acute and chronic stress, respectively (36). Oxidative stress produces a large amount of damaged proteins, which may lead to overloading of the autophagosomal system and result in a reduction in autophagic activity $(36,37)$. In the high-glucose conditions of Müller cells, autophagic machinery is triggered, although autophagic flux is compromised. The autophagic substrate p62 accumulates in the cytosol, signaling apoptosis and extensive VEGF production (38). The level of LC3II/I is an indicator of the initiation of autophagy. It has been well demonstrated that autophagy depends on the activity of Atg5, Atg7 and Atg12. Of all these indicators, Beclin1 is required for Atg-7-dependent autophagy (22). The results of the present study demonstrated that the autophagic flux was weakened in the retinas of the OIR mice. As increased protein aggregation may contribute to inflammasome activation and tissue injury, reduced autophagy and increased inflammation may be involved in angiogenesis $(39,40)$.

ROS act as a 'double-edged sword' in the vasculature. In wound healing, physiological angiogenesis is induced by tissue hypoxia and ROS, resulting in the production of VEGF $(41,42)$. However, the supply of oxygen and nutrients caused by pathological angiogenesis results in the uncontrolled generation of new blood vessels, which leads to an abnormal vascular pattern (43). The present study revealed that RNV was generated via HIF-1 $\alpha /$ VEGF-A signaling. Hypoxia stimulated the accumulation of ROS, which was mediated by NOX, and increased pyroptosis through activation of the NLRP3-caspase-1-IL-1 $\beta$ pathway, while autophagic activity was compromised in this process.
A recent study demonstrated that impaired autophagy induces pro-inflammatory and pro-angiogenic proteins, which results in angiogenesis (39). Autophagic dysfunction and oxidative stress are implicated in retinitis pigmentosa (44). Mild oxidative stress triggers cell survival and repair mechanisms, including the autophagic pathway. However, in the case of severe oxidative stress, excessive levels of ROS for a prolonged period leads to oxidative damage and, ultimately, cell death (45). The results of certain studies suggest that pyroptosis may be functionally associated with autophagy. Inhibition of autophagy stimulates pneumococcus-induced pyroptosis and protects microglial cells against pyroptosis (33). The present study demonstrated, for the first time to the best of our knowledge, that ROS and pyroptosis are activated and autophagy is compromised in OIR mice, suggesting that severe oxidative stress induces upregulation of pyroptosis and inhibition of autophagy. The combination of oxidative stress, pyroptosis and impaired autophagy may serve an important role in the pathophysiology of OIR. A possible limitation of the present study was that only the phenomena of oxidative stress, autophagy, and pyroptosis were investigated; the mechanisms accounting for their association remain unclear, and alterations in apoptosis were not detected. Further investigation is warranted to illustrate these changes in retinal neovascularization.

\section{Acknowledgements}

Not applicable.

\section{Funding}

The present study was supported by Natural Foundation of Liaoning Province (grant nos. 2015E21SF005 and 20180550740).

\section{Availability of data and materials}

The datasets used and/or analyzed during the current study are available from the corresponding author on reasonable request.

\section{Authors' contributions}

SW and L-YJ performed the research. L-YJ, SW and LL analyzed the data. SW, L-YJ and J-ML conceived and designed the research. J-ML wrote the manuscript.

\section{Ethics approval and consent to participate}

All investigations were approved by the Animal Care and Use Committee of Dalian Medical University and conformed to the US National Institutes of Health Guide for the Care and Use of Laboratory and the ARRIVE guidelines.

\section{Patient consent for publication}

Not applicable.

\section{Competing interests}

The authors declare that they have no competing interests. 


\section{References}

1. Kim YW and Byzova TV: Oxidative stress in angiogenesis and vascular disease. Blood 123: 625-631, 2014.

2. Bhatt L, Groeger G, McDermott K and Cotter TG: Rod and cone photoreceptor cells produce ROS in response to stress in a live retinal explant system. Mol Vis 16: 283-293, 2010.

3. Al-Shabrawey M, Bartoli M, El-Remessy AB, Platt DH, Matragoon S, Behzadian MA, Caldwell RW and Caldwell RB: Inhibition of $\mathrm{NAD}(\mathrm{P}) \mathrm{H}$ oxidase activity blocks vascular endothelial growth factor overexpression and neovascularization during ischemic retinopathy. Am J Pathol 167: 599-607, 2005.

4. Guo C, Yang M, Jing L, Wang J, Yu Y, Li Y, Duan J, Zhou X, Li Y and Sun Z: Amorphous silica nanoparticles trigger vascular endothelial cell injury through apoptosis and autophagy via reactive oxygen species-mediated MAPK/Bcl-2 and PI3K/Akt/mTOR signaling. Int J Nanomed 11: 5257-5276, 2016.

5. Adamis AP and Shima DT: The role of vascular endothelial growth factor in ocular health and disease. Retina 25: 111-118, 2005.

6. Zepeda AB, Pessoa A Jr, Castillo RL, Figueroa CA, Pulgar VM and Farías JG: Cellular and molecular mechanisms in the hypoxic tissue: Role of HIF-1 and ROS. Cell Biochem Funct 31: 451-459, 2013

7. Saito S, Lin YC, Tsai MH, Lin CS, Murayama Y, Sato R and Yokoyama KK: Emerging roles of hypoxia-inducible factors and reactive oxygen species in cancer and pluripotent stem cells. Kaohsiung J Med Sci 31: 279-286, 2015.

8. Kietzmann $\mathrm{T}$ and Görlach A: Reactive oxygen species in the control of hypoxia-inducible factor-mediated gene expression. Semin Cell Dev Biol 16: 474-486, 2005.

9. Green DR, Galluzzi L and Kroemer G: Mitochondria and the autophagy-inflammation-cell death axis in organismal aging. Science 333: 1109-1112, 2011.

10. Vande Walle L and Lamkanfi M: Pyroptosis. Curr Biol 26: R568-R572, 2016.

11. Man SM, Karki R and Kanneganti TD: Molecular mechanisms and functions of pyroptosis, inflammatory caspases and inflammasomes in infectious diseases. Immunol Rev 277: 61-75, 2017.

12. Li L, Tan J, Miao Y, Lei P and Zhang Q: ROS and autophagy: Interactions and molecular regulatory mechanisms. Cell Mol Neurobiol 35: 615-621, 2015.

13. Levonen AL, Hill BG, Kansanen E, Zhang J and Darley-Usmar VM: Redox regulation of antioxidants, autophagy, and the response to stress: Implications for electrophile therapeutics. Free Radic Biol Med 71: 196-207, 2014.

14. Lapaquette P, Guzzo J, Bretillon L and Bringer MA: Cellular and molecular connections between autophagy and inflammation. Mediators Inflamm 2015: 398483, 2015.

15. Blasiak J, Petrovski G, Veréb Z, Facskó A and Kaarniranta K: Oxidative stress, hypoxia, and autophagy in the neovascular processes of age-related macular degeneration. Biomed Res Int 2014: 768026, 2014.

16. Grossniklaus HE, Kang SJ and Berglin L: Animal models of choroidal and retinal neovascularization. Prog Retin Eye Res 29: 500-519, 2010.

17. Kilkenny C, Browne WJ, Cuthill IC, Emerson $M$ and Altman DG: Improving bioscience research reporting: The ARRIVE guidelines for reporting animal research. PLoS Biol 8: e1000412, 2010

18. Livak KJ and Schmittgen TD: Analysis of relative gene expression data using real-time quantitative PCR and the 2(-Delta Delta C(T)) method. Methods 25: 402-408, 2001.

19. Wilkinson-Berka JL, Deliyanti D, Rana I, Miller AG, Agrotis A, Armani R, Szyndralewiez C, Wingler K, Touyz RM, Cooper ME, et al: NADPH oxidase, NOX1, mediates vascular injury in ischemic retinopathy. Antioxid Redox Signal 20: 2726-2740, 2014

20. Li J, Wang JJ, Yu Q, Chen K, Mahadev K and Zhang SX: Inhibition of reactive oxygen species by Lovastatin downregulates vascular endothelial growth factor expression and ameliorates blood-retinal barrier breakdown in $\mathrm{db} / \mathrm{db}$ mice: Role of NADPH oxidase 4. Diabetes 59: 1528-1538, 2010.

21. Harris J: Autophagy and cytokines. Cytokine 56: 140-144, 2011.

22. Klionsky DJ, Abdelmohsen K, Abe A, Abedin MJ, Abeliovich H, Acevedo Arozena A, Adachi H, Adams CM, Adams PD, Adeli K, et al: Guidelines for the use and interpretation of assays for monitoring autophagy (3rd edition). Autophagy 12: 1-222, 2016.

23. Zin A and Gole GA: Retinopathy of prematurity-incidence today. Clin Perinatol 40: 185-200, 2013.
24. Gilbert C, Fielder A, Gordillo L, Quinn G, Semiglia R, Visintin P and Zin A; International NO-ROP Group: Characteristics of infants with severe retinopathy of prematurity in countries with low, moderate, and high levels of development: Implications for screening programs. Pediatrics 115: e518-e525, 2005.

25. Cabral T, Mello LGM, Lima LH, Polido J, Regatieri CV, Belfort R Jr and Mahajan VB: Retinal and choroidal angiogenesis: A review of new targets. Int J Retina Vitreous 3: 31, 2017.

26. Cervia D, Catalani E, Dal Monte M and Casini G: Vascular endothelial growth factor in the ischemic retina and its regulation by somatostatin. J Neurochem 120: 818-829, 2012.

27. Finkel T and Holbrook NJ: Oxidants, oxidative stress and the biology of ageing. Nature 408: 239-247, 2000.

28. Gu Q, He Y, Ji J, Yao Y, Shen W, Luo J, Zhu W, Cao H, Geng Y, $\mathrm{Xu}$ J, et al: Hypoxia-inducible factor $1 \alpha(\mathrm{HIF}-1 \alpha)$ and reactive oxygen species (ROS) mediates radiation-induced invasiveness through the SDF-1 $\alpha /$ CXCR4 pathway in non-small cell lung carcinoma cells. Oncotarget 6: 10893-10907, 2015

29. Andrei C, Dazzi C, Lotti L, Torrisi MR, Chimini G and Rubartelli A: The secretory route of the leaderless protein interleukin 1beta involves exocytosis of endolysosome-related vesicles. Mol Biol Cell 10: 1463-1475, 1999.

30. Miao EA, Rajan JV and Aderem A: Caspase-1-induced pyroptotic cell death. Immunol Rev 243: 206-214, 2011.

31. Zhou R, Tardivel A, Thorens B, Choi I and Tschopp J: Thioredoxin-interacting protein links oxidative stress to inflammasome activation. Nat Immunol 11: 136-140, 2009.

32. Wu D, Han R, Deng S, Liu T, Zhang T, Xie H and Xu Y: Protective effects of flagellin A N/C against radiation-induced NLR pyrin domain containing 3 inflammasome-dependent pyroptosis in intestinal cells. Int J Radiat Oncol Biol Phys 101: 107-117, 2018.

33. Kim JY, Paton JC, Briles DE, Rhee DK and Pyo S: Streptococcus pneumoniae induces pyroptosis through the regulation of autophagy in murine microglia. Oncotarget 6: 44161-44178, 2015.

34. Szumiel I: Autophagy, reactive oxygen species and the fate of mammalian cells. Free Radic Res 45: 253-265, 2011.

35. Filomeni G, Desideri E, Cardaci S, Rotilio G and Ciriolo MR: Under the ROS...thiol network is the principal suspect for autophagy commitment. Autophagy 6: 999-1005, 2014.

36. Mitter SK, Song C, Qi X, Mao H, Rao H, Akin D, Lewin A, Grant M, Dunn W Jr, Ding J, et al: Dysregulated autophagy in the RPE is associated with increased susceptibility to oxidative stress and AMD. Autophagy 10: 1989-2005, 2014.

37. Kaarniranta K, Sinha D, Blasiak J, Kauppinen A, Veréb Z, Salminen A, Boulton ME and Petrovski G: Autophagy and heterophagy dysregulation leads to retinal pigment epithelium dysfunction and development of age-related macular degeneration. Autophagy 9: 973-984, 2013.

38. Lopes de Faria JM, Duarte DA, Montemurro C, Papadimitriou A, Consonni SR and Lopes de Faria JB: Defective autophagy in diabetic retinopathy. Invest Ophthalmol Vis Sci 57: 4356-4366, 2016.

39. Liu J, Copland DA, Theodoropoulou S, Chiu HA, Barba MD, Mak KW, Mack M, Nicholson LB and Dick AD: Impairing autophagy in retinal pigment epithelium leads to inflammasome activation and enhanced macrophage-mediated angiogenesis. Sci Rep 6: 20639, 2016.

40. Wang Y, Hanus J, Abu-Asab M, Shen D, Ogilvy A, Ou J, Chu XK, Shi G, Li W, Wang S and Chan CC: NLRP3 upregulation in retinal pigment epithelium in age-related macular degeneration. Int J Mol Sci 17: pii: E73, 2016.

41. Sen CK, Khanna S, Babior BM, Hunt TK, Ellison EC and Roy S: Oxidant-induced vascular endothelial growth factor expression in human keratinocytes and cutaneous wound healing. J Biol Chem 277: 33284-33290, 2002.

42. Schreml S, Szeimies RM, Prantl L, Karrer S, Landthaler M and Babilas P: Oxygen in acute and chronic wound healing. Br J Dermatol 163: 257-268, 2010

43. Chung AS and Ferrara N: Developmental and Pathological Angiogenesis. Annu Rev Cell Dev Biol 27: 563-584, 2011.

44. Moreno ML, Mérida S, Bosch-Morell F, Miranda M and Villar VM: Autophagy dysfunction and oxidative stress, two related mechanisms implicated in retinitis pigmentosa. Front Physiol 9: 1008, 2018.

45. Sureshbabu A, Ryter SW and Choi ME: Oxidative stress and autophagy: Crucial modulators of kidney injury. Redox Biol 4: 208-214, 2015

This work is licensed under a Creative Commons Attribution-NonCommercial-NoDerivatives 4.0 International (CC BY-NC-ND 4.0) License. 